en fonction du "poids optique ", ont été déterminées pour des échantillons de caséinate de calcium du lait écrémé dont le chauffage préliminaire avait été fait à des températures différentes.

$2^{\circ} \mathrm{La}$ majorité du matériel a un diamètre de moins de $200 \mathrm{~m} \mu$ avec une dimension moyenne d'environ $90 \mathrm{~m} \mu$.

$3^{\circ}$ Une petite quantité de matériel à particules plus grandes, probablement du phosphate de calcium colloïdal et de grands agrégats de caséinate de calcium, est également présente.

$4^{\circ}$ Le chauffage préliminaire jusqu'à $95^{\circ} \mathrm{C}$. a peu d'influence sur la courbe de distribution des dimensions des particules.

\title{
LES BESOINS EN LAIT DE L'ENFANT ET LES QUANTITÉS MINIMA NÉCESSAIRES A SA CROISSANCE (1)
}

\author{
par \\ M. L. RIBADEAU-DÚMAS \\ Médecin du Service maternel et infantile de la Salpètrière.
}

Les innombrables recherches concernant la valeur biologique des aliments ont confirmé l'observation séculaire qui avait mis en évidence la supériorité du lait sur toutes les formules alimentaires, quelles qu'elles soient.

Le lait représente un mélange harmonieux et complet des substances utiles à la croissance de l'organisme jeune. Les variantes de sa composition répondent seulement aux besoins des espèces. On a toujours pensé qu'au nourrisson humain convenait le lait de sa mère. Certaines circonstances économiques ou sociales conduisent à d'autres modes d'allaitement, généralement par le lait de vache. Celui-ci présente de grandes analogies avec le lait de femme, sans toutefois lui être tout à fait superposable : il nécessite done, pour être adapté aux facultés digestives du nourrisson, une correction qui est connue et universellement appliquée.

Mais, s'il est exceptionnel d'observer chez l'enfant nourri convenablement par sa mère, un trouble de croissance ayant quelque gravité, il n'en est pas de même pour l'enfant nourri au lait de vache. Il y a, en effet, entre le lait de vache et le lait de femme une différence que la clinique certifie, mais que l'analyse biologique n'a pas encore suffisamment précisée pour qu'on puisse, par une modification appropriée, tirer de l'allaitement artificiel les mêmes bienfaits que de l'allaitement naturel.

D'une manière générale, l'enfant normal qui est soumis à un allaitement convenablement dirigé, progresse régulièrement. Mieux

(1) Paru dans le Volume jubilaire du Prolesseur Ch. Porcher. 
encore, l'enfant-type présente une croissance admirable avec une alimentation souvent paradoxale. Par contre, un très grand nombre d'enfants souffrent de l'allaitement artificiel et présentent des dystrophies d'origine alimentaire plus ou moins graves. On a pu incriminer, dans les cas de ce genre, une erreur dans la préparation du lait, la mauvaise qualité ou une composition anormale du lait par mancuvre industrielle, falsification, carence quelconque; le fait est possible. Une "mutation lactée ", suivant l'expression de M. VARIOT, peut être suivie de suecès. Mais il ne manque pas d'observations où, toutes les précautions étant prises et le lait irréprochable, l'enfant présente des troubles digestifs contre lesquels échouent toutes les mesures ayant pour objet de modifier le lait qui lui est destiné. Le tube digestif de l'enfant est inadapté à l'allaitement artificiel : tôt ou tard, il présentera des troubles alimentaires. La raison en est peut-être à une " méiopagie " des épithéliums digestifs, peut-être à une anomalie cellulaire plus générale encore, dépendant d'une hérédité malencontreuse.

Les difficultés de l'allaitement artificiel ont été reconnues de tous temps. Au dix-huitième siècle, Deskssarts pensait déjà que dans certaines circonstances, le lait se comportait comme un poison.

C'est pour cette raison qu'établissant une règle commune, beaucoup d'observateurs, à l'exemple de CzERny et de FEER, limitent la ration lactée de l'enfant à la dose maximum de 500 ou $600 \mathrm{gr}$., le surplus calorique nécessaire étant fourni par des aliments dont le choix est basé sur le principe de l'isodynamie. L'alimentation fondamentale est complétée par l'alimentation complémentaire, dont l'une des indications est l'apport de vitamines. Malgré toutes les précautions prises, il peut arriver que l'enfant supporte mal le lait, même à doses limitées. Il s'agit alors d'une intolérance passagère ou durable liée à un état pathologique, congénital ou acquis. Ces faits ont suscité un très grand nombre d'études qui, avec HAMBURGER, Epstein, Hindes, Jundell, Moll et Stranksy, Klincke, Stolte, ont abouti à l'institution des régimes sans lait ou pauvres en lait.

On trouve dans la thèse de M. VErmorel, faite sous l'inspiration de M. Cathala, en 1929, une relation détaillée des méthodes employées par ces auteurs, plus ou moins dérivées des recherches d'Osborne et Mendel sur les régimes artificiels. Les protéines sont fournies par le foie, la viande, le ris de veau ; les hydrates de carbone, par les farines et le sucre ; les graisses, par les jaunes d'œuf, l'huilede foie de morue, les huiles végétales ; les sels, par le mélange d'OsBorns et MENDEL. Cette alimentation est utilisée dès l'âge de trois mois, pendant un mois, quelquefois 300 jours et plus. Elle est réservée aux enfants atteints de tétanie, de pyloro-spasme, de dystrophies alimentaires, anorexies, anémies alimentaires, eczémas. Elle serait bien 
tolérée par l'enfant, mais le gain pondéral est faible. HAMBURGER admet d'ailleurs que l'accroissement en poids est un test de peu de valeur, bien inférieur à l'amélioration de l'état général, le développement neuro-moteur, la composition du sang, la résistance aux infections.

Avec MM. Mathieu et WiLlemin, nous avons cherché à simplifier ces régimes, et nous avons utilisé, comme protéines, les protéines végétales, en particulier l'aleurone de tournesol déshuilé, tel qu'il a été préparé par M. ANDRé. Nous avons constitué avec des farines, du sucre, des sels et l'aleurone, une alimentation dont la composition donne le même chiffre de calories que le lait. Nous n'avons pas tardé à constater expérimentalement qu'un tel régime uniquement basé sur l'isodynamie était insuffisant, et que, très rapidement, il fallait y ajouter les substances qui sont qualitativement nécessaires à la croissance, telles que l'huile de foie de morue et la levure de bière.

Quel que soit le régime sans lait adopté, on ne saurait le continuer très longtemps, et d'ailleurs beaucoup d'enfants ne tardent pas à témoigner une répugnance légitime pour une alimentation artificielle, même consciencieusement calculée. Le lait donne certainement un développement plus harmonieux qu'un régime sans lait. Celui-ci, de toute évidence, doit être réservé aux cas d'intolérance par le lait, et cela pour un temps limité. En réalité, il est très rare qu'un enfant ne puisse supporter le lait au moins à doses limitées. L'adjonction d'une petite quantité de cet aliment à des régimes purement artificiels, améliore considérablement la croissance. C'est là une observation qui a pu être aisément faite en clinique par M. EPstEIn et par nousmême.

Cette constatation est d'ailleurs tout à fait en rapport avec les faits expérimentaux recueillis par MM. HoPkINs, KENNEDY et Dutcher, Buissex et Golding, et avec les observations prises chez les enfants par Corry Maun.

Le problème qui se pose pour les nourrissons qui paraissent ne pas tolérer le lait, n'est donc pas de les alimenter sans lait, mais de leur faire prendre avec un régime artificiel, la dose de lait nécessaire et suffisante pour leur assurer un développement satisfaisant.

A côté des régimes sans lait, il y a donc place pour les régimes lactés restreints, dont la supériorité est évidente.

Nous avons étudié ces régimes lactés restreints avec MM. René Mathind, Willemin et Mile Doméla, dont la thèse contient tous les détails expérimentaux et cliniques concernant ce travail (1930).

L'alimentation de base est représentée par une bouillie de crème de riz, maltée, sucrée et salée, complétée en ce qui concerne les protéines par l'aleurone de tournesol ou le soja. La valeur calorique d'une telle bouillie est de 700 calories au litre. 
Expérimentée d'abord chez le rat, cette bouillie a été donnée ensuite à des nourrissons présentant, malgré des mutations lactées fréquentes, une dystrophie progressive par alimentation au lait de vache.

Pendant un temps variable, la bouillie assure une croissance à peu près normale ; à la troisième semaine en moyenne, on observe chez le nourrisson un blocage de la courbe de poids.

Nous nous sommes demandé si la croissance serait plús régulière en ajoutant au régime de l'enfant les graisses qui sont à un taux extrêmement réduit dans la bouillie réalisée. Les graisses choisies ont été la graisse du beurre, de l'huile de foie de morue ou du lait de femme centrifugé. Les doses ont varié de 10 à $30 \mathrm{gr}$.; l'effet n'a presque jamais été favorable, toujours insuffisant. On était en droit de penser à une insuffisance qualitative des protéines végétales. En augmentant les protéines aux dépens des hydrates de carbone, on constitue une ration plus riche en calories d'origine protéique et en acides aminés. En pareil cas, l'augmentation de poids devient plus régulière, mais le dêséquilibre de la ration ne tarde pas à provoquer des troubles digestifs.

Un autre essai est tenté avec la levure de bière. La raison qui nous incite à ce choix est, d'une part, la richesse de ce produit en acides aminés et, d'autre part, sa forte teneur en vitamines $B$ et $\mathbf{B}^{\prime}$. On en donne 2 à $3 \mathrm{gr}$. : la courbe est immédiatement débloquée. Elle devient normale lorsqu'il y a addition d'une petite dose d'huile de foie de morue $\left(2 \mathrm{~cm}^{3}\right)$.

On peut done conclure qu'un apport suffisant de vitamines au régime, lui confère une haute valeur qualitative, et la courbe de poids, à nouveau, va remonter régulièrement. Mais l'accroissement se ralentit peu à peu et va cesser dans un très grand nombre de cas d'être satisfaisant, soit au bout de 2 à 3 mois.

En ajoutant à l'aliment de base une certaine quantité de lait, l'effet est bien plus constant.

Il s'agit de déterminer :

$1^{0}$ si l'addition de lait au régime améliore à elle seule la courbe de poids ;

$2^{\circ}$ si la quantité de lait nécessaire peut être fixée ;

$3^{\circ}$ à quel élément du lait l'activation de la croissance est due.

Mile Doméća a entrepris une série d'expériences à ce point de vue, en procédant d'abord à une série d'essais sur le rat.

Le régime de base accepté par l'animal est le régime-type : 
Tournesol $\ldots \ldots \ldots \ldots \ldots \ldots \ldots \ldots \ldots \ldots$

$16 \mathrm{gr}$.

Crème de riz $\ldots \ldots \ldots \ldots \ldots \ldots \ldots \ldots$.

$80 \mathrm{gr}$.

Lactate de calcium ................ 3 gr.

Chlorure de sodium $. \ldots \ldots \ldots \ldots \ldots \ldots \ldots, \quad 1$ gr.

$100 \mathrm{gr}$.

On introduit dans le régime du rat de la poudre de lait demiécrémée, les quantités de tournesol et de crème de riz étant proportionnellement réduites, pour que la valeur calorique du mélange ne soit pas modifiée. Les différentes doses de lait ont été de $2,4,5,10 \%$.

On conclut :

a) qu'une faible quantité de lait mélangé au régime améliore nettement la courbe de poids et augmente la eroissance ;

b) que la quantité nécessaire et suffisante pour obtenir ce résultat est de 5 gr. de poudre de lait pour 100 gr. de ration;

c) que des doses supérieures ne sont pas favorables;

d) que le lait mélangé au régime, améliorant la croissance, ne donne jamais une courbe de poids absolument normale dans nos expériences. Cependant l'apport d'huile de foie de morue et de vitamine $\mathrm{B}$ donne des résultats favorables.

L'observation clinique autorise à des conclusions de même ordre, moins catégoriques toutefois, parce qu'il existe ici de nombreux facteurs individuels et pathologiques. Il est possible de fixer un minimum de lait favorisant la croissance ; il est plus difficile d'en fixer un maximum. Du premier point de vue, la dose nécessaire paraît répondre au dixième de la ration; au delà, l'effet obtenu est des plus variables. Par exemple, un enfant prenant en lait $10 \%$ du régime, accuse des troubles sensibles dès qu'on dépasse cette dose. D'autres nourrissons auront une belle courbe de croissance avec une quantité de lait égale à 15,20 ou $30 \%$ de la ration. Des remarques de même ordre peuvent être faites avec des laits de composition différente (lait condensé, lait frais). Ici, l'adjonetion de graisse n'est favorable que si l'enfant est capable de la digérer.

D'autre part, le régime lacté restreint comportera toujours l'addition des différentes vitamines. Quoi qu'il en soit, un fait d'observation se dégage très nettement de nos expériences : on peut alimenter un enfant avec un régime lacté restreint; des prises de lait même peu importantes confèrent à l'enfant un aspect de bonne santé, alors qu'il reste pâle, en général, avec le régime végétarien sans lait, malgré une quantité suffisante de vitamine $B$. Un régime absolument privé de lait peut avoir ses indications, mais jusqu'à présent, nous n'avons pas observé d'enfant soumis au régime précité qui fût totalement intolérant au lait. L'anaphylaxie au lait est, en effet, exceptionnelle. 
Il est ainsi prouvé une fois de plus, que le nourrisson ne peut se passer de lait qu'à grand'peine ; c'est son aliment spécifique, et il importe d'introduire dans son régime une certaine quantité de lait, même minime. Le fait curieux est la disproportion entre les doses employées et l'effet obtenu : il semble que si le lait peut cesser d'être un aliment d'entretien, il reste indispensable comme aliment de croissance.

Reste à savoir quel est l'élément du lait qui confère à cet aliment une supériorité aussi évidente. Nous ne pouvons donner à cette question une réponse précise ; la méthode analytique ne nous permet pas de conclure.

L'adjonction au régime sans lait des lactalbumines mises à notre disposition par M. Penau ne nous donne aucun résultat, pas plus que la caséine; des essais faits avec le mélange salin d'OsBorne et MENDEL n'ont été suivis d'aucun succès. De même avec les sucres de lait.

Le rôle des graisses est peut-être plus important : si à un régime sans lait, donné au rat, on ajoute $9 \%$ de graisse de beurre, l'état général de l'animal se trouve amélioré ; la croissance est un peu meilleure avec $12 \%$ de graisse de beurre. Elle devient très satisfaisante avec l'addition de levure de bière. Chez l'enfant, étant donné les états pathologiques très divers devant lesquels on se trouve, les résultats sont très variables. Les graisses ajoutées isolément au régime de base, même les graisses de lait de femme, ne donnent que très rarement satisfaction. Par contre, le lait complet assure une croissance naturellement plus régulière et plus belle que le lait écrémé, à un même taux de calories. Il faut donc, en pratique, surveiller les progrès de la tolérance de l'enfant pour le lait, et lui donner du lait complet aussitôt que cela est possible.

En ce qui concerne les vitamines, nous avons toujours constaté qu'un régime sans lait devait comporter toutes les vitamines, et cela à un taux élevé. Si chacune d'elles est nécessaire à une croissance irréprochable, il importe de signaler le rôle de premier plan du facteur B d'utilisation des glucides (RANDOIN et SIMONNET) : on ne saurait élever le poids de l'enfant avec un régime artificiel sans lui adjoindre une forte quantité de facteur B. Cette remarque vaut même dans le cas où, au régime, on ajoute une certaine quantité de lait : la bouillie tournesolée additionnée de vitamine $B$ ne donne pas de croissance assurée, la bouillie tournesolée et lactée non plus, mais une bouillie lactée et riche en vitamine $B$ donne les meilleurs résultats. On ne saurait en être étonné si l'on tient compte du fait que la quantité de lait ajoutée au régime est toujours médiocre.

Toutefois, l'expérience actuelle enseigne que même le lait de femme, ou le lait de vache, est très souvent pauvre en vitamine B, 
en raison d'habitudes alimentaires défectueuses, de telle sorte que bien souvent en elinique on est obligé de faire prendre à un enfant soumis à l'alimentation lactée intégrale de la vitamine $\mathbf{B}$ sous quelque forme que ce soit, pour triompher d'un arrêt de croissance parfois inexplicable.

Nous n'avons done pas pu démontrer l'existence dans le lait d'une substance assurant la croissance. Il est bien probable qu'il s'agit d'un complexe alimentaire, dont les éléments merveilleusement équilibrés concourent par leur ensemble à une nutrition parfaite de l'organisme jeune.

\section{EN RÉSUMÉ :}

On peut alimenter un enfant avee un régime sans lait, tout au moins à partir de trois mois. Mais la croissance reste imparfaite.

Un régime lacté restreint donne des résultats nettement supérieurs. Il semble que l'enfant ne puisse se passer de son aliment spécifique, et comme l'écrit Mlle DoмéLA, même dans le cas de dyspepsie de lait, alors que cet aliment détermine à haute dose, des troubles digestifs et des dystrophies, il semble qu'il existe une dose parfaitement supportée par le nourrisson et indispensable à sa croissance.

L'intolérance totale au lait est dans le jeune âge extrêmement rare.

Il semble qu'il existe pour l'enfant dyspeptique un seuil au-dessous duquel les quantités de lait données sont insuffisantes, et au-dessus duquel elles sont nuisibles. La dose de lait minimum acceptée par l'enfant et assurant sa croissance, représente le dixième de sa ration, telle que nous l'avons étudiée avec un aliment toujours le même : la bouillie tournesolée. La clinique permet d'ailleurs d'augmenter ces doses variables suivant les cas et la progression de la tolérance au lait de l'enfant.

Nous n'avons pu déterminer quel était l'élément du lait indispensable à la croissance. Mais il est probable que toutes les substances constitutives du lait y contribuent. En tout cas, dans la composition d'un régime lacté restreint, il paraît indiqué de faire intervenir un lait gras complet, sans oublier les besoins de l'organisme en vitamine $B$. 